\title{
Diffusion Tensor Imaging Reveals Whole-Brain Microstructural Changes in the P301 L Mouse Model of Tauopathy
}

\author{
Aidana Massalimova ${ }^{\mathrm{a}}$ Ruiqing $\mathrm{Ni}^{\mathrm{a}}$ Roger M. Nitsch ${ }^{\mathrm{b}, \mathrm{c}}$ Marco Reisert ${ }^{\mathrm{d}}$ \\ Dominik von Elverfeldt ${ }^{d}$ Jan Klohs ${ }^{a, b}$ \\ anstitute for Biomedical Engineering, ETH \& University of Zurich, Zurich, Switzerland; b Zurich Neuroscience Center \\ (ZNZ), Zurich, Switzerland; Institute for Regenerative Medicine, University of Zurich, Schlieren, Switzerland; \\ ${ }^{\mathrm{d} D e p a r t m e n t}$ of Radiology, Medical Physics, Medical Center - University of Freiburg, Faculty of Medicine, University \\ of Freiburg, Freiburg, Germany
}

\section{Keywords}

Magnetic resonance imaging · Diffusion tensor imaging ·

Tau · Frontotemporal dementia - Animal models

\begin{abstract}
Introduction: Increased expression of hyperphosphorylated tau and the formation of neurofibrillary tangles are associated with neuronal loss and white matter damage. Using high-resolution ex vivo diffusion tensor imaging (DTI), we investigated microstructural changes in the white and grey matter in the P301L mouse model of human tauopathy at 8.5 months of age. For unbiased computational analysis, we implemented a pipeline for voxel-based analysis (VBA) and atlas-based analysis (ABA) of DTI mouse brain data. Methods: Hemizygous and homozygous transgenic P301L mice and non-transgenic littermates were used. DTI data were acquired for generation of fractional anisotropy (FA), mean diffusivity (MD), radial diffusivity (RD), and axial diffusivity (AD) maps. VBA on the entire brain was performed using SPM8 and the SPM Mouse toolbox. Initially, all DTI maps were coregistered with the Allen mouse brain atlas to bring them to one common coordinate space. In VBA, coregistered DTI maps were normalized and smoothed in order to perform
\end{abstract}

two-sample and unpaired $t$ tests with false discovery rate correction to compare hemizygotes with non-transgenic littermates, homozygotes with non-transgenic littermates, and hemizygotes with homozygotes on each DTI parameter map. In $A B A$, the average values for selected regions of interests were computed with coregistered DTI maps and labels in Allen mouse brain atlas. Afterwards, a Kruskal-Wallis oneway ANOVA on ranks with a Tukey post hoc test was executed on the estimated average values. Results: With VBA, we found pronounced and brain-wide spread changes when comparing homozygous, P301L mice with non-transgenic littermates, which were not seen when comparing hemizygous P301 L with non-transgenic animals. Statistical comparison of DTI metrics in selected brain regions by ABA corroborated findings from VBA. FA was found to be decreased in most brain regions, while $M D, R D$, and $A D$ were increased in homozygotes compared to hemizygotes and non-transgenic littermates. Discussion/Conclusion: High-resolution ex vivo DTI demonstrated brain-wide microstructural and gene-dose-dependent changes in the P301L mouse model of human tauopathy. The DTI analysis pipeline may serve for the phenotyping of models of tauopathy and other brain diseases.

(c) 2021 The Author(s)

Published by S. Karger AG, Basel karger@karger.com www.karger.com/ndd

Karger $\stackrel{\text { ' }}{5}$

GOPEN ACCESS
(C) 2021 The Author(s)

Published by S. Karger AG, Basel

This is an Open Access article licensed under the Creative Commons Attribution-NonCommercial-4.0 International License (CC BY-NC) (http://www.karger.com/Services/OpenAccessLicense), applicable to the online version of the article only. Usage and distribution for commercial purposes requires written permission.
Correspondence to:

Jan Klohs, klohs@biomed.ee.ethz.ch 


\section{Introduction}

Alzheimer's disease (AD), progressive supranuclear palsy, and corticobasal degeneration and frontotemporal dementia including frontotemporal dementia with Parkinsonism linked to chromosome 17 (FTDP-17) and Pick's disease are characterized by the progressive formation of neurofibrillary tangles (NFTs) [1,2]. NFTs are intracellular neuronal lesions composed of insoluble, conformationally abnormal, hyperphosphorylated accumulations of tau in neurons and glia [2]. The presence of NFTs in the brain triggers a complex cascade of biochemical and cellular processes that result in neurodegeneration and neuronal loss [2]. The occurrence of NFTs correlates well with spatial patterns of neuronal loss and is related to the degree of cognitive deficits $[3,4]$.

Magnetic resonance imaging (MRI) has been used to non-invasively characterize tissue changes in the brain associated with tau pathology. Structural MRI studies have shown that NFT deposition is associated with grey matter atrophy that results from neurodegeneration and that different tauopathies display distinctive regional patterns of grey matter atrophy [5-8]. In addition, white matter changes such as white matter atrophy and the occurrence of white matter hyperintensities have been reported [9]. Such observations are indicative of white matter damage and demyelination caused by axonal tau pathology [10]. Despite their usefulness, structural MRI provides little information about the underlying neurodegenerative changes in the brain.

Diffusion-weighted imaging is a MRI technique that probes the directional diffusivity of water molecules and can, thus, yield information about the microstructural properties of brain tissue [11-13]. Diffusion imaging data are commonly analysed using a tensor model, that is, by applying diffusion tensor imaging (DTI) [14]. DTI shows sensitivity to axon and myelin pathology, where breakdown of white matter integrity results in measurable differences in diffusion of water molecules $[15,16]$. DTI has been widely used to assess white matter abnormalities related to tau pathologies [17-23]. A few DTI studies have also reported changes in diffusivity in the grey matter [24, 25]. Importantly, DTI microstructural changes seem to precede gross anatomical changes seen on structural MRI [24].

Genetic studies show that the development of neurodegenerative tauopathies is associated with missense mutations in the microtubule-associated tau protein (MAPT) [26]. Based on this finding, transgenic mouse lines with MAPT mutations gene including P301S [27] and P301L [28-31] have been developed. Some models have the advantage of tetracycline-controlled transcriptional suppression of tau [31]. Histological studies have demonstrated deposition of NFTs pathology, in particular, in the cortex (CRX) and hippocampus and marked atrophy of these areas [27-32]. Behavioural analysis revealed cognitive deficits in learning and motor tasks [30, 31].

Transgenic mouse models of tauopathy have been used in MRI studies [33-38]. Previous DTI studies assessing microstructural changes in $\mathrm{rTg} 4510$ mice, which express transgenic tau P301L mutations driven by a $\mathrm{Ca}^{2+}$ / calmodulin kinase II promoter system $[30,31]$ in the white and grey matter produced conflicting results [3336]. Thus, we wished to determine DTI metrics in transgenic P301L mice, which express tau under the control of the murine Thy 1.2 promoter $[28,29]$. In the present study, we used ex vivo DTI to assess microstructural changes in 8.5-month-old P301L mice in both white and grey matter regions. We leveraged the ability of long data acquisitions that are possible in ex vivo DTI to achieve a high spatial resolution, without suffering from image corruption due to motion or breathing that are common in in vivo DTI $[12,13]$. We implemented a pipeline for voxel-based analysis (VBA) and atlas-based analysis (ABA) for unbiased computational analysis of DTI mouse brain data. To test for gene-dose-dependent effects, we assessed both hemizygous and homozygous P301L mice and compared them with non-transgenic littermates. We hypothesized that effects on DTI microstructural parameters would be stronger in homozygotes than in hemizygotes because of the stronger tau transgene expression in homozygous P301L mice.

\section{Materials and Methods}

\section{Animal Models}

$\mathrm{P} 301 \mathrm{~L}$ mice, expressing the human 4 repeat tau isoform under the control of the murine Thy 1.2 promoter, were backcrossed with C57BL/ 6 mice for $>20$ generations and maintained on a C57BL/6 background (B6.Dg-Tg [Thy1.2-TauP301L] 183 Nitsch) $[28,29]$. Hemizygous P301L mice were used for breeding to generate homozygous P301L mice. Hemizygous ( $n=8 ; 5$ females $/ 3$ males) and homozygous $(n=8 ; 5$ females $/ 3$ males) P301L mice of 8.5 months of age and age-matched nontransgenic littermates ( $n=8 ; 2$ females/6 males) were used. Animals were housed in ventilated cages inside a temperature-controlled room, under a 12-h dark/light cycle. Each cage housed up to 5 mice. Paper tissue and red Tecniplast mouse house ${ }^{\circledR}$ (Tecniplast, Milan, Italy) shelters were placed in cages as environmental enrichments. Pelleted food (3437PXL15, CARGILL) and water were provided ad libitum. 
Magnetic Resonance Imaging

Mice were intracardially perfused under deep anaesthesia of ketamine/xylazine/acepromazine maleate $(75 / 10 / 2 \mathrm{mg} / \mathrm{kg}$ body weight, i.p. bolus injection) with $10 \mathrm{~mL}$ ice-cold $0.1 \mathrm{M}$ PBS (pH 7.4 ) and $4 \%$ paraformaldehyde in $0.1 \mathrm{M}$ PBS. Heads were removed and post-fixed in paraformaldehyde solution for 6 days. Thereafter, samples were stored in $0.1 \mathrm{M} \mathrm{PBS}\left(\mathrm{pH} \mathrm{7.4)}\right.$ ) at $4^{\circ} \mathrm{C}$ until measurements. Brains were not removed from the skull, which has been shown previously to preserve cortical and central brain structure [35]. The heads were placed in a $15 \mathrm{~mL}$ centrifuge tube filled with perfluoropolyether (Fomblin Y, LVAC 16/6, average molecular weight 2,700, Sigma-Aldrich, St. Louis, MO, USA). Data were acquired on a small-animal MRI system (BioSpec 94/30 animal MRI system, Bruker Corporation) at $400 \mathrm{MHz}$ and equipped with a BGA $12 \mathrm{AS}$ HP gradient system with a maximum gradient strength of $400 \mathrm{mT} / \mathrm{m}$ and minimum rise time of $70 \mu$ s. A cryogenic $2 \times 2$ channel radiofrequency surface coil probe (overall coil size $20 \times 27 \mathrm{~mm}^{2}$, Bruker BioSpin AG, Fällanden, Switzerland) with the coil system operating at $30 \mathrm{~K}$ (Bruker BioSpin AG, Fällanden, Switzerland) was used in combination with a circularly polarized $86-\mathrm{mm}$ volume resonator for transmission.

DTI was acquired with 3D multishot echo-planar imaging sequence ( 4 shots) with a field of view of $18 \times 12 \times 9 \mathrm{~mm}$ and a matrix dimension of $180 \times 120 \times 90$, resulting in a nominal voxel resolution of $100 \times 100 \times 100 \mu \mathrm{m}$. The following imaging parameters were chosen: a repetition time of $1,500 \mathrm{~ms}$, an echo time of $28 \mathrm{~ms}$, no averaging, 5 volumes acquired with $a b$-values of 0 (A0), and a $b$-value of $4,000 \mathrm{~s} / \mathrm{mm}^{2}$ along 60 diffusion encoding directions. A global and MAPSHIM protocol with a field map (default settings) was used for shimming. The total acquisition time per brain was 9 h $45 \mathrm{~min}$.

\section{Image Processing}

Image processing was performed with the Nora medical imaging platform (http://www.nora-imaging.com/; University Medical Center Freiburg, Germany) [39, 40]. All the data were first uploaded to the platform and automatically converted into NIFTi format. Diffusion tensors were estimated using DTI\&Fibertools (https://www.uniklinik-freiburg.de/mr-en/research-groups/diffperf/fibertools.html), and on this basis, fractional anisotropy (FA), mean diffusivity (MD), radial diffusivity (RD), and axial diffusivity (AD) maps were computed. To normalize images to an atlas space, SPM8 (Wellcome Trust Centre for Neuroimaging, UCL, UK) together with the SPM Mouse toolbox (University of Oxford, UK) were used. For each scan, the b0 image was normalized to the mouse atlas space using the SPM normalize and segment tool. Further, the Allen mouse brain atlas (http://mouse.brain-map.org/ static/atlas) was also non-linearly registered to the atlas space to enable the VBA and ABA. Maximization of the mutual information was used for image registration. Mutual information or relative entropy is a widely used similarity measure in medical image registration [41]. As we maximize the mutual information, the information from the combined images is minimized compared to 2 separate images. All considered microstructural maps (FA, MD, $\mathrm{RD}$, and $\mathrm{AD}$ ) were finally warped to atlas space for VBA and ABA. To evaluate if the registration was done properly, we visually inspected images after registration with SPM, overlaying one image on the top of another. Registration for VBA and ABA was found to be done properly.

DTI P301L Mouse Model
Voxel-Based Analysis

VBA on the entire brain was performed using SPM8. In addition, the SPM Mouse toolbox (University of Oxford, UK) was used because it extends the SPM functionality to the animal brains. All normalized images were smoothed $\left(0.1 \times 0.1 \times 0.1 \mathrm{~mm}^{2}\right.$ Gaussian $)$. Besides, the average maps of each parameter for every subgroup were generated to visualize the estimated t-values on it. In total, 3 unpaired two-sample $t$ tests were executed, including the comparisons of hemizygotes versus homozygotes, hemizygotes versus nontransgenic littermates, and homozygotes versus non-transgenic littermates. Statistical maps were thresholded with a significance level of $p<0.001$ and then, were corrected using false discovery rate. Results were superimposed on FA, MD, RD, and AD maps.

\section{Atlas-Based Analysis}

Allen mouse brain atlas was overlaid on each normalized image. The atlas contains 670 labelled regions. In order to limit the number of candidate regions for the examination, we selected region of interests (ROIs) for the anterior commissure (AC), corpus callosum (CC), cerebral peduncle (CPD), cortex (CRX), hippocampal region (HPR), subiculum (SUB), and thalamus (TH). ROIs were slightly eroded to avoid partial volume effects. Then, the average values of each ROI from the atlas were computed. ROI data were tested using the Kolmogorov-Smirnov test, which showed that all data were not normally distributed. Variance homogeneity was tested using the Levene test which showed regions with and without homogeneous variance. Thus, a Kruskal-Wallis one-way ANOVA on ranks was used to determine differences between $A D$, $\mathrm{MD}, \mathrm{RD}$, and FA in these ROIs. A Tukey post hoc test was used for pairwise multiple comparisons between hemizygotes versus homozygotes, hemizygotes versus non-transgenic littermates, and homozygotes versus non-transgenic littermates. We used $p<0.05$, $p<0.01, p<0.001$, and $p<0.0001$ as our significance levels. All computations were done using Python version 3.5.0.

\section{Results}

The reconstructed data are available in the Zenodo repository (DOI: 10.5281/zenodo.4005562). We also made the code for VBA and ABA available (https://github.com/ aidanamv/ABA-pipeline).

We examined ex vivo DTI metrics of the brains of hemizygous and homozygous P301L mice at 8.5 months of age. Resulting maps of the $\mathrm{FA}, \mathrm{MD}, \mathrm{RD}$, and $\mathrm{AD}$ are shown in Figure 1 and in different views in online supplementary Figures 1-4 (for all online suppl. material, see www.karger.com/doi/10.1159/000515754). The highresolution FA maps show clearly white matter structures that are highly anisotropic, like the CC and the AC and hippocampal commissure (HC).

\section{VBA Reveals Gene-Dose-Dependent Effects on DTI} Metrics

VBA was performed to compare differences in FA, $\mathrm{MD}, \mathrm{RD}$, and $\mathrm{AD}$ between groups and to test for gene- 


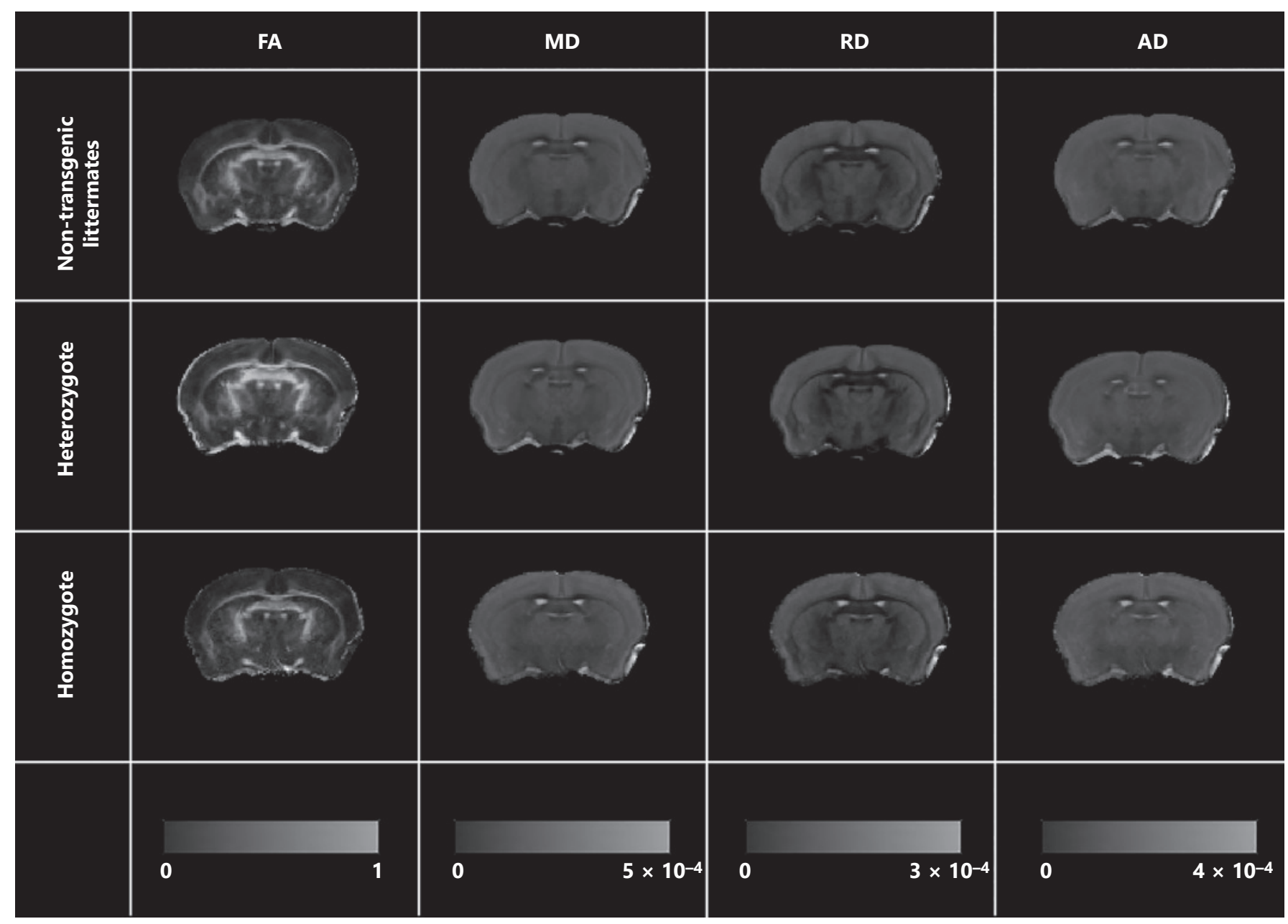

Fig. 1. Representative coronal $\mathrm{FA}, \mathrm{MD}, \mathrm{RD}$, and $\mathrm{AD}$ maps of 8.5-month-old non-transgenic littermates, hemizygous, and homozygous P301L mice. Shown are views at approximately -1.8 $\mathrm{mm}$ from bregma. $\mathrm{AD}, \mathrm{RD}$, and $\mathrm{MD}$ values are in units of $\times 10^{-4}$ $\mathrm{mm}^{2} / \mathrm{s}$. FA, fractional anisotropy; $\mathrm{MD}$, mean diffusivity; RD, radial diffusivity; $\mathrm{AD}$, axial diffusivity. dose-dependent effects. Firstly, we compared hemizygous P301L mice with non-transgenic littermates, but no different changes between the 2 groups were observed. Secondly, we compared homozygous P301L mice with non-transgenic littermates (Fig. 2). The colour gradient represents the magnitude of significant differences between groups (unpaired two-sample $t$ test with clusterwise FDR correction). Pronounced changes in the white matter such as the CC and $\mathrm{HC}$ were observed for all DTI metrics. In addition, group differences were also detected in grey matter structures such as the CRX, hippocampus, and TH. Thirdly, we compared hemizygotes with homozygotes (Fig. 3). Group differences were seen in both the grey and white matter in all DTI metrics in the HC only.

\section{ABA Reveals Grey and White Matter Alterations Related to Tau Pathology}

Results of ABA are shown in Table 1 and Figure 4. Statistical analysis was performed in 3 white matter regions (AC, CC, and CPD) and 4 grey matter regions (HPRs, CRX, SUB, and TH).

\section{Fractional Anisotropy}

In the $\mathrm{AC}$, there was no difference in $\mathrm{FA}$ between groups observed. In the CC, HPR, SUB, and TH, FA values were significantly decreased in homozygous P301L mice compared to hemizygous transgenic mice and compared to non-transgenic littermates. In the CPD and CRX, FA values were found reduced in hemizygote P301L mice compared to non-transgenic controls. 


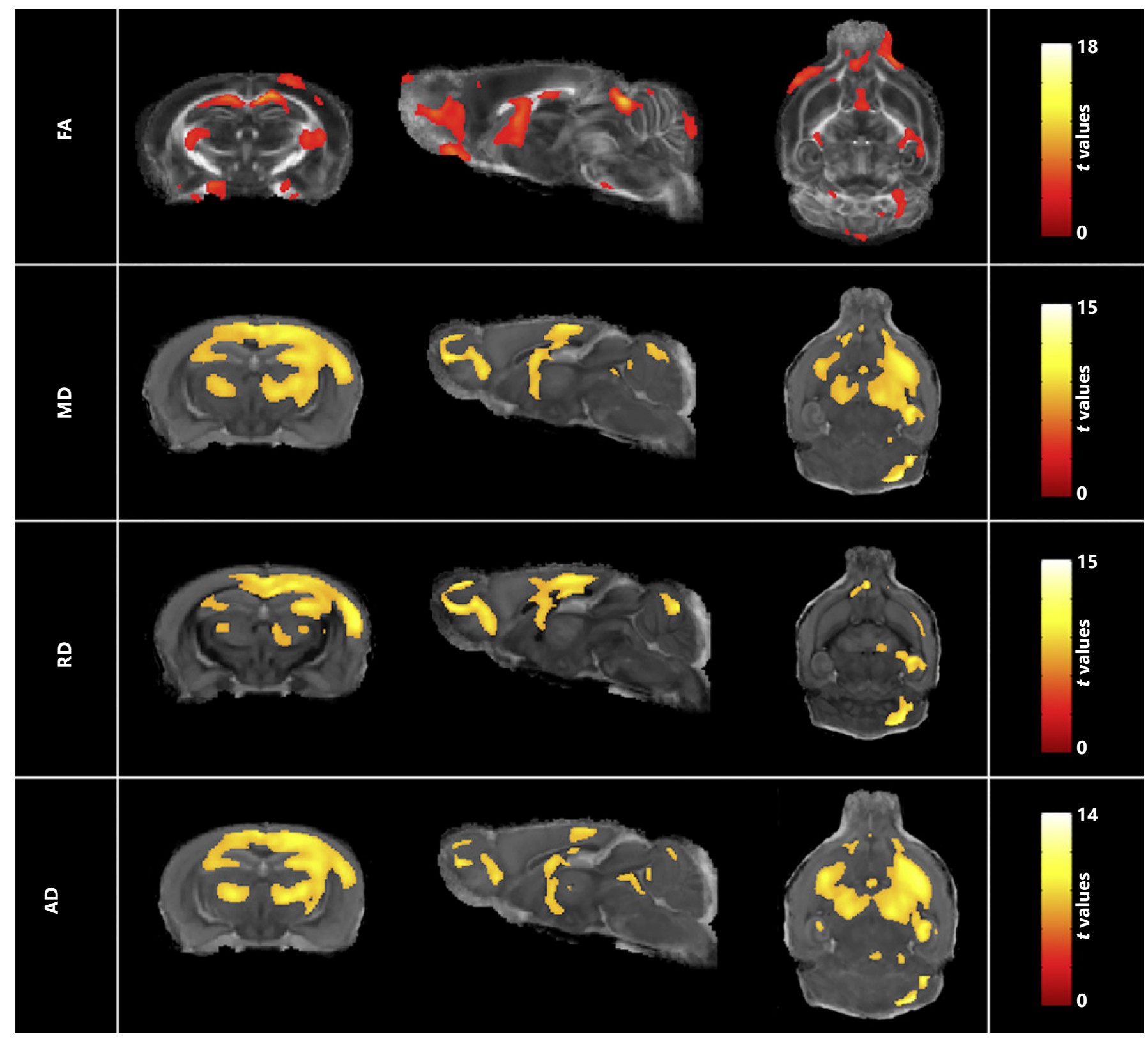

Fig. 2. VBA ( $t$ values overlaid on mouse brain template) comparing homozygous P301L mice with non-transgenic littermates (unpaired two-sample $t$ test $p<0.001$, clusterwise FDR correction). Changes in DTI metrics were observed across the brain, in both

Mean Diffusivity

$M D$ values were significantly increased in the AC, CC, CPD, CRX, HPR, and TH in homozygote P301L mice compared to non-transgenic and hemizygous littermates. For the SUB, values were higher in homozygotes than in nontransgenic littermates. In the CPD, MD values were higher in hemizygous P301L mice and non-transgenic controls.

DTI P301L Mouse Model grey and white matter areas. VBA, voxel-based analysis; FDR, false discovery rate; DTI, diffusion tensor imaging; FA, fractional anisotropy; $\mathrm{MD}$, mean diffusivity; $\mathrm{RD}$, radial diffusivity; $\mathrm{AD}$, axial diffusivity.

\section{Radial Diffusivity}

$R D$ values were significantly increased in the AC, CC, CPD, HPR, SUB, and TH in homozygous P310L mice compared to hemizygotes and non-transgenic littermates. In the CRX, RD values were found significantly increased in homozygous P301L mice compared to hemizygotes. Only in the CPD, RD values were significantly 


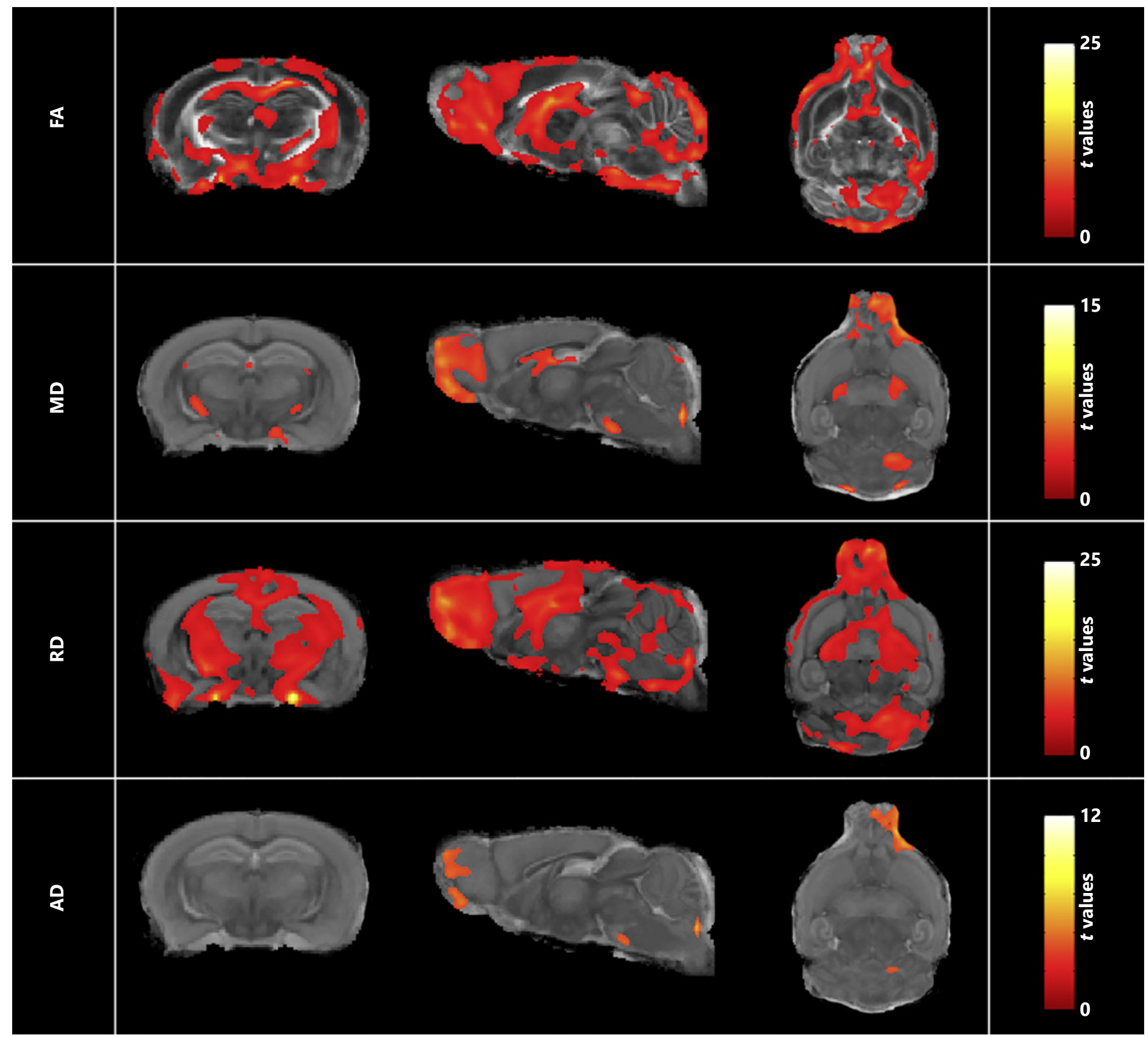

Fig. 3. Grade of tau gene expression determines severity of DT alterations. VBA ( $t$ values overlaid on mouse brain template) comparing hemizygous with homozygous P301L mice $(p<0.001$, clusterwise FDR correction). Changes in DTI metrics were observed across the brain. VBA, voxel-based analysis; FDR, false discovery rate; DTI, diffusion tensor imaging; FA, fractional anisotropy; $\mathrm{MD}$, mean diffusivity; $\mathrm{RD}$, radial diffusivity; $\mathrm{AD}$, axial diffusivity. increased in hemizygous P301L mice compared to nontransgenic littermates.

\section{Axial Diffusivity}

$A D$ values were significantly increased in the $A C, C C$, CPD, CRX, HPR, and TH in homozygous P301L mice compared to non-transgenic littermates. In the AC, CPD, $\mathrm{CRX}, \mathrm{HPR}$, and $\mathrm{TH}, \mathrm{AD}$ values were also significantly higher in homozygous P301L mice than in hemizygous mice. No differences were observed between hemizygous P301L mice and non-transgenic littermates in all regions tested. 


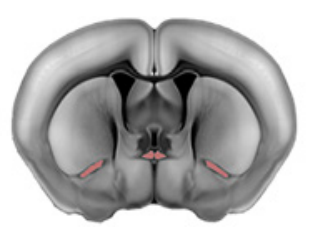

Anterior commissure (AC)

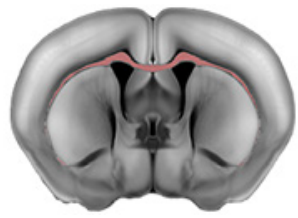

Corpus callosum (CC)

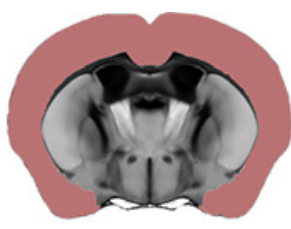

Cortex (CRX)

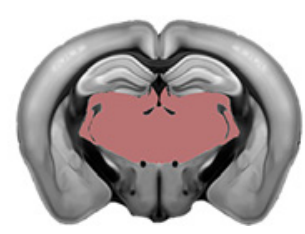

Thalamus $(\mathrm{TH})$

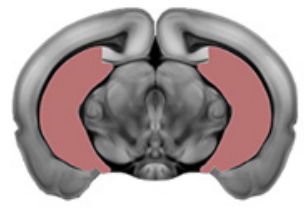

Hippocampal region (HPR)

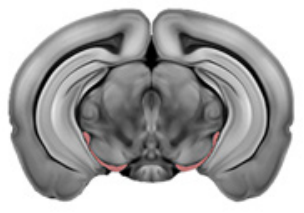

Cerebral peduncle (CPD)

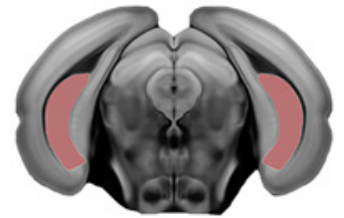

Subiculum (SUB)
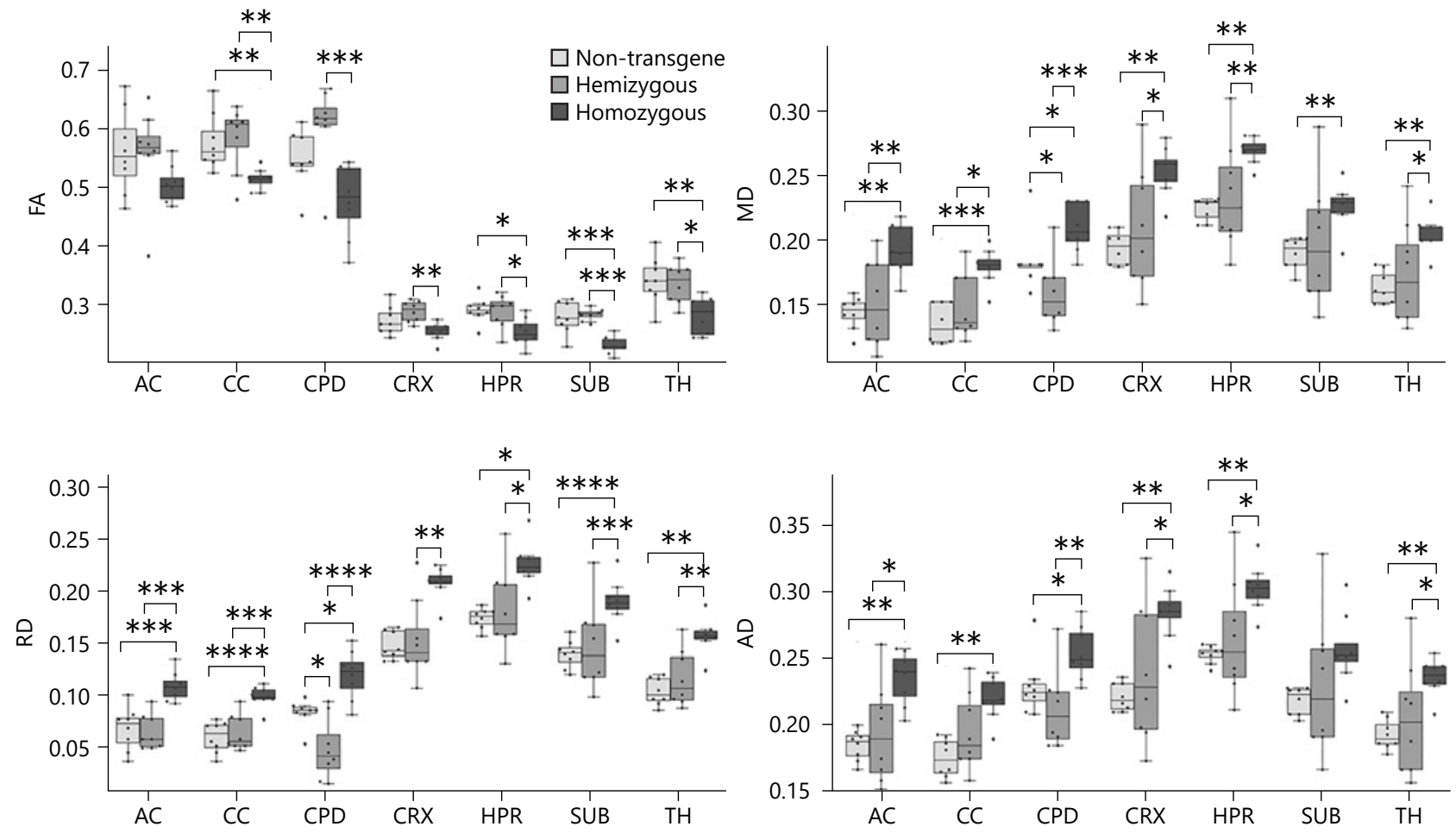

Fig. 4. Gene-dose-dependent effects on DTI metrics. ABA of selected brain regions in non-transgenic littermates, hemizygous, and homozygous P301L mice. Regions analysed where the AC, CC, CPD, CRX, HPR, SUB, and TH. Data are represented in box plots. Centre lines show the median; box limits indicate the first and third quartile; whiskers show the minimum and maximum. One-

\section{Discussion/Conclusion}

DTI has been previously used in patient studies to assess microstructural changes related to tau pathologies [17-25]. Application of DTI to animal models of tauopa- way ANOVA. ${ }^{*} p<0.05,{ }^{* *} p<0.01,{ }^{* * *} p<0.001$, and ${ }^{* * * *} p<$ 0.0001 . DTI, diffusion tensor imaging; $\mathrm{ABA}$, atlas-based analysis; $\mathrm{AC}$, anterior commissure; CC, corpus callosum; CPD, cerebral peduncle; CRX, cerebral cortex; HPR, hippocampal region; SUB, subiculum; TH, thalamus; FA, fractional anisotropy; MD, mean diffusivity; $\mathrm{RD}$, radial diffusivity; $\mathrm{AD}$, axial diffusivity. 
Table 1. DTI parameters for different regions from ABA

\begin{tabular}{|c|c|c|c|c|}
\hline Region & $\begin{array}{l}\text { DTI } \\
\text { parameter }\end{array}$ & $\begin{array}{l}\text { Non-transgenic } \\
\text { littermates } \\
(n=8)\end{array}$ & $\begin{array}{l}\text { Hemizygous } \\
\text { P301L } \\
(n=8)\end{array}$ & $\begin{array}{l}\text { Homozygous } \\
\text { P301L } \\
(n=8)\end{array}$ \\
\hline $\mathrm{AC}$ & $\begin{array}{l}\text { FA } \\
\text { MD } \\
\text { RD } \\
\text { AD }\end{array}$ & $\begin{array}{l}0.56 \pm 0.07 \\
0.14 \pm 0.01 \\
0.06 \pm 0.02 \\
0.18 \pm 0.02\end{array}$ & $\begin{array}{l}0.56 \pm 0.08 \\
0.15 \pm 0.03 \\
0.06 \pm 0.02 \\
0.19 \pm 0.03\end{array}$ & $\begin{array}{l}0.50 \pm 0.03 \\
0.19 \pm 0.02^{*, \#} \\
0.11 \pm 0.01^{*, \#} \\
0.23 \pm 0.02^{*, \#}\end{array}$ \\
\hline $\mathrm{CC}$ & $\begin{array}{l}\mathrm{FA} \\
\mathrm{MD} \\
\mathrm{RD} \\
\mathrm{AD}\end{array}$ & $\begin{array}{l}0.58 \pm 0.05 \\
0.14 \pm 0.02 \\
0.06 \pm 0.01 \\
0.18 \pm 0.01\end{array}$ & $\begin{array}{l}0.58 \pm 0.05 \\
0.15 \pm 0.03 \\
0.06 \pm 0.02 \\
0.19 \pm 0.03\end{array}$ & $\begin{array}{l}0.51 \pm 0.02^{*, \#} \\
0.18 \pm 0.01^{*, \#} \\
0.10 \pm 0.01^{*, \#} \\
0.22 \pm 0.02^{*}\end{array}$ \\
\hline CPD & $\begin{array}{l}\mathrm{FA} \\
\mathrm{MD} \\
\mathrm{RD} \\
\mathrm{AD}\end{array}$ & $\begin{array}{l}0.55 \pm 0.05 \\
0.18 \pm 0.02 \\
0.08 \pm 0.01 \\
0.23 \pm 0.00\end{array}$ & $\begin{array}{l}0.61 \pm 0.07 \\
0.16 \pm 0.03^{*} \\
0.05 \pm 0.03^{*} \\
0.21 \pm 0.02\end{array}$ & $\begin{array}{l}0.48 \pm 0.06^{\#} \\
0.21 \pm 0.02^{*, \#} \\
0.12 \pm 0.02^{*, \#} \\
0.25 \pm 0.01^{*, \#}\end{array}$ \\
\hline CRX & $\begin{array}{l}\text { FA } \\
\mathrm{MD} \\
\mathrm{RD} \\
\mathrm{AD}\end{array}$ & $\begin{array}{l}0.28 \pm 0.02 \\
0.19 \pm 0.01 \\
0.15 \pm 0.01 \\
0.22 \pm 0.00\end{array}$ & $\begin{array}{l}0.29 \pm 0.02 \\
0.21 \pm 0.05 \\
0.15 \pm 0.04 \\
0.24 \pm 0.05\end{array}$ & $\begin{array}{l}0.26 \pm 0.02^{\#} \\
0.25 \pm 0.02^{*, \#} \\
0.21 \pm 0.02^{\#} \\
0.28 \pm 0.02^{*, \#}\end{array}$ \\
\hline HPR & $\begin{array}{l}\mathrm{FA} \\
\mathrm{MD} \\
\mathrm{RD} \\
\mathrm{AD}\end{array}$ & $\begin{array}{l}0.29 \pm 0.02 \\
0.22 \pm 0.01 \\
0.17 \pm 0.01 \\
0.25 \pm 0.00\end{array}$ & $\begin{array}{l}0.29 \pm 0.03 \\
0.23 \pm 0.04 \\
0.18 \pm 0.04 \\
0.26 \pm 0.04\end{array}$ & $\begin{array}{l}0.26 \pm 0.02^{*, \#} \\
0.27 \pm 0.01^{*, \#} \\
0.23 \pm 0.02^{*, \#} \\
0.30 \pm 0.02^{*, \#}\end{array}$ \\
\hline SUB & $\begin{array}{l}\mathrm{FA} \\
\mathrm{MD} \\
\mathrm{RD} \\
\mathrm{AD}\end{array}$ & $\begin{array}{l}0.28 \pm 0.03 \\
0.19 \pm 0.01 \\
0.10 \pm 0.01 \\
0.22 \pm 0.01\end{array}$ & $\begin{array}{l}0.28 \pm 0.00 \\
0.20 \pm 0.05 \\
0.12 \pm 0.04 \\
0.23 \pm 0.04\end{array}$ & $\begin{array}{l}0.23 \pm 0.01^{*, \#} \\
0.23 \pm 0.0202^{*} \\
0.16 \pm 0.02^{*, \#} \\
0.26 \pm 0.02\end{array}$ \\
\hline $\mathrm{TH}$ & $\begin{array}{l}\text { FA } \\
\text { MD } \\
\text { RD } \\
\text { AD }\end{array}$ & $\begin{array}{l}0.34 \pm 0.04 \\
0.16 \pm 0.01 \\
0.12 \pm 0.01 \\
0.19 \pm 0.01\end{array}$ & $\begin{array}{l}0.34 \pm 0.03 \\
0.17 \pm 0.04 \\
0.14 \pm 0.03 \\
0.20 \pm 0.04\end{array}$ & $\begin{array}{l}0.28 \pm 0.03^{*, \#} \\
0.21 \pm 0.01^{*, \#} \\
0.13 \pm 0.02^{*, \#} \\
0.24 \pm 0.02^{*, \#}\end{array}$ \\
\hline
\end{tabular}

Mean $\pm \mathrm{SD} ; \mathrm{AD}, \mathrm{RD}$, and MD values are in units of $\times 10^{-3} \mathrm{~mm}^{2} / \mathrm{s}$. DTI, diffusion tensor imaging; $\mathrm{ABA}$, atlasbased analysis; AC, anterior commissure; CC, corpus callosum; CPD, cerebral peduncle; CRX, cerebral cortex; HPR, hippocampal region; SUB, subiculum; TH, thalamus; $\mathrm{MD}$, mean diffusivity; RD, radial diffusivity; $\mathrm{AD}$, axial diffusivity. ${ }^{*} p<0.05$ versus non-transgenic littermates. ${ }^{*} p<0.05$ versus hemizygous. One-way ANOVA.

on DTI metrics in the P301L models of human tauopathy. We acquired ex vivo DTI data in 8.5-month-old hemizygous and homozygous P301L mice and age-matched non-transgenic littermates. VBA revealed microstructural changes in both white and grey matter regions in homozygotes but not in hemizygotes $\mathrm{P} 301 \mathrm{~L}$ mice. ABA showed that decreased FA in most brain regions, while $\mathrm{MD}, \mathrm{RD}$, and $\mathrm{AD}$ were increased in homozygous $\mathrm{P} 301 \mathrm{~L}$ mice compared to hemizygous and non-transgenic littermates.
In contrast to previous studies where DTI in mice was performed in vivo [33-36], we performed ex vivo DTI. Compared to the in vivo setting, ex vivo DTI has the advantage that it is not hampered by motion artefacts, for example, breathing. It also allows for prolonged scanning times. We acquired 3D data with $100 \mu \mathrm{m}$ isotropic resolution in $9 \mathrm{~h} 45 \mathrm{~min}$. We measured complete head samples, which preserve the structural integrity of the brains [35] in a high-field small-bore magnet with cryogenic radiofrequency coils [42] to obtain diffusion data with suffi- 
cient signal-to-noise ratio for automated VBA and ABA analyses. Nevertheless, ex vivo DTI requires chemical fixation with formaldehyde to preserve the tissue and the procedure alters the macroscopic and microscopic properties of the tissue. For example, a study by Ma et al. [43] has shown that most of the white structures demonstrated significantly larger ex vivo volumes after fixation than in vivo volumes (i.e., internal capsule and fimbria) except for the smallest white matter structure (e.g., AC), which was significantly smaller ex vivo. Conversely, grey matter structures (e.g., neocortex, TH, and hippocampus) decreased in volume. Fixation does alter the magnitude of water diffusion in the tissue, but measures of the directionality of diffusion are less affected [44-46]. The subsequent placement of samples in PBS removed excess fixative and, while it does not reverse the chemical effects of fixation, it has been shown to partially restore diffusivity of the tissue [46]. As samples are equally treated, the volume changes induced by fixation do not hamper advanced, unbiased computational approaches and allow detection of anatomical phenotypes in transgenic animals. The reduced diffusivity of fixed tissue requires strong gradients for strong diffusion weighting. These are commonly available in small-bore animal scanners. We used a b-value of $4,000 \mathrm{~s} / \mathrm{mm}^{2}$, as recommended for ex vivo DTI [47].

Previous studies have used ROI analysis [33-36], while we opted for VBA and ABA for statistical analysis of DTI data. For ROI analysis, selected areas are manually drawn, which is more time consuming, requires a priori anatomical localization, and is prone to bias [48]. Moreover, in many neurodegenerative diseases, microstructural changes occur across the whole brain, and conventional ROI analysis may miss to detect changes in areas that are not selected [49]. VBA has been widely used in structural MRI as an unbiased approach to assess morphological changes of tissue $[43,50]$. Voxel-by-voxel statistical comparison is performed once all brain images are normalized to a template space, with the assumption that each individual voxel represents the same anatomical location of the brain $[49,51,52]$.

The spatial normalization of the brains in VBA was based on scalar image-based registration, incorporating 12-parameter affine transformation and diffeomorphic mapping, which is essentially done to match internal brain structures to the template. One may apply a lowpass filter to smooth normalized images; however, the fact, if the particular location is the same voxel in other images, is still questionable due to the potential atrophy in the brain [51]. Smoothing in DTI is also challenging due to thin tracts of white matter and heterogeneity of the FA maps. Nevertheless, this method is still in use in biomedical imaging due to its simplicity [50]. Besides, its statistical power is poor due to the high level of noise.

With VBA, we found marked changes when comparing homozygous P301L mice with non-transgenic littermates, while we did not observe differences between hemizygous transgenic mice and non-transgenic littermates. The comparisons demonstrated that changes in DTI metrics are gene-dose dependent, where mice with the stronger tau transgene expression (i.e., homozygous P301L mice) show the phenotype. This finding is in line with the results of the ABA where differences in DTI metrics were found between homozygous P301L mice and homozygous and non-transgenic littermates. It may be speculated that use of larger group sizes or animals of older ages might have also revealed differences between hemizygous P301L and non-transgenic animals, as the hemizygous mice also have tau pathology.

While the VBA revealed areas of significant changes in DTI metrics, it does not inform about the direction of the changes. Thus, we used ABA to obtain regional values of $\mathrm{FA}, \mathrm{MD}, \mathrm{RD}$, and $\mathrm{AD}$. ABA analysis is based on automatic segmentation of brain regions defined in the atlas after normalization [51-54]. This provides high sensitivity to small and widely distributed changes, but it fails when the regions are not within the anatomical boundaries of the predefined atlas [54]. Results from ABA revealed a decrease in FA and increased $M D, R D$, and $A D$ values in the CC compared to non-transgenic littermates, while other white matter regions were unchanged. An earlier study by Sahara et al. reported age-dependent changes in DTI metrics in rTg4510 mice [33]. A decrease in FA values was noted in the CC, AC, internal capsule, splenium, and fimbria of 8-month-old rTg4510 mice. RD was found increased in the CC, splenium, and fimbria, while $\mathrm{MD}$ and $\mathrm{AD}$ were unchanged. A study by Colgan et al. [36] found lower FA values and higher MD in the CC of 8.5-month-old rTg4510 mice than in non-transgenic littermates. A study by Wells et al. reported increased RD values in the CC in $\mathrm{rTg} 4510$, while $\mathrm{FA}, \mathrm{MD}$, and $\mathrm{AD}$ were not different from non-transgenic littermates [34].

DTI is also increasingly used to assess microstructural changes in the grey matter [55]. In our study, we found decreased FA values and increased $M D, R D$, and $A D$ values in the HPRs, CRX, and TH in homozygous P301L mice compared to non-transgenic controls. A previous study by Wells et al. [34] reported increased FA and MD in the CRX and hippocampus of rTg4510 mice. In the TH, the MD was found increased, while the FA was un- 
changed. A study by Holmes et al. [35] reported increased FA and MD in the CRX and hippocampus of rTg4510 mice. In the TH, FA was increased, while MD was not different. In a study by Colgan et al. [36], MD was increased in the hippocampus and CRX. FA was higher in the hippocampus but was not different in the CRX. No change in FA and MD has been observed in the TH.

Differences in DTI metrics in cross-sectional studies in transgenic mouse models of tauopathy based on MAPT mutations may arise due to the use of different strains, that is, the type of tau mutation, promoter of transgene expression, transgene expression levels, genetic background, and housing conditions [56], which are known to affect the phenotype. Furthermore, methodological differences in DTI data acquisition (in vivo vs. ex vivo and sequence parameters) and different analysis methods (ROI, VBA, ABA etc.) may contribute to the observed differences in studies. We made $\mathrm{FA}, \mathrm{MD}, \mathrm{RD}$, and $\mathrm{AD}$ maps and analysis code publicly available, which enable other groups to perform an independent analysis with our data, to validate the code, and to enable of image-based meta-analysis. Finding converging patterns of microstructural changes or identifying different phenotypes between different tau mouse models compared to clinical studies would increase the translational value of preclinical DTI studies. In this regard, the observed pattern of decreased FA and increased MD, RD, and $\mathrm{AD}$ values in P301L mice in the current study are in good agreement with DTI changes observed in human tauopathies such as progressive supranuclear palsy [21], frontotemporal dementia [22], mild cognitive impairment [24], and Alzheimer's disease [18, 25].

Effects of tauopathy on DTI metrics in the grey and white matter are complex and may involve axonal damage, myelin injury, neuronal degeneration, and gliosis, which all have been found in tauopathies $[17,18,23,25$, $28,29,33,38,57]$. The interpretation of changes in the measured diffusion tensor is thus awaiting histological determination. Furthermore, future studies may seek to examine microstructural changes in vivo in a longitudinally designed study. For example, it would be important to determine the onset of changes in DTI and how this is related to gross anatomical changes and cognitive function. Studies in patients with mild cognitive impairment have shown that changes in DTI metrics in the hippocampus, a region involved in working memory formation can predict cognitive decline and constitute a more sensitive predictor than changes in hippocampal volume [24]. Given the limitations of DTI, more advanced models for reconstruction diffusion MRI data may be explored [12, 36, $58]$.
In summary, ex vivo DTI demonstrated brain-wide and gene-dose-dependent microstructural changes in the P301L mouse model of human tauopathy, similar to what has been observed in patients with tauopathies. The DTI analysis pipeline may serve for the phenotyping of models of tauopathy and other brain diseases.

\section{Acknowledgements}

The authors acknowledge Daniel Schuppli at the Institute for Regenerative Medicine, University of Zurich, for technical assistance. We thank Dr. Sandra Iglesias and Prof. Klaas Enno Stephan (Translational Neuromodeling Unit, University of Zurich and ETH Zurich) for help with the VBA.

\section{Statement of Ethics}

All the experiments were performed in accordance with the Swiss Federal Act on Animal Protection and were approved by the Cantonal Veterinary Office, Zurich (permit number: ZH082/18). All procedures fulfilled the ARRIVE guidelines on reporting animal experiments.

\section{Conflict of Interest Statement}

J.K. is an associate editor of Neurodegenerative Diseases. All the other authors have no conflicts of interest to declare.

\section{Funding Sources}

J.K. received funding from the Swiss National Science Foundation (320030_179277), in the framework of ERA-NET NEURON (32NE30_173678/1), the Synapsis Foundation, the Olga Mayenfisch Stiftung, and the Vontobel Foundation. R.N. received funding from the Synapsis Foundation career development award (2017 CDA-03).

\section{Author Contributions}

This study was conceived and designed by R.N. and J.K. R.M.N. provided the P301L mouse model. M.R. and D.v.E. provided the software platform for DTI reconstruction. Material preparation, data collection, and analysis were performed by A.M., R.N., and M.R. The first draft of the manuscript was written by A.M. and J.K. All the authors commented on the previous versions of the manuscript. All the authors read and approved the final manuscript. 


\section{References}

1 Iqbal K, Alonso AC, Chen S, Chohan MO, ElAkkad E, Gong CX, et al. Tau pathology in Alzheimer disease and other tauopathies. Biochim Biophys Acta. 2005;1739(2-3):198-210.

2 Dugger BN, Dickson DW. Pathology of neurodegenerative diseases. Cold Spring Harb Perspect Biol. 2017;9(7):a028035.

3 Joie RLa, Visani AV, Baker SL, Brown JA, Bourakova V, Cha J, et al. Prospective longitudinal atrophy in Alzheimer's disease correlates with the intensity and topography of baseline tau-PET. Sci Transl Med. 2020; 12(524):1-13.

4 Iaccarino L, Tammewar G, Ayakta N, Baker SL, Bejanin A, Boxer AL, et al. Local and distant relationships between amyloid, tau and neurodegeneration in Alzheimer's disease. NeuroImage Clin. 2018;17(February 2017): 452-64.

5 Gröschel K, Hauser TK, Luft A, Patronas N, Dichgans J, Litvan I, et al. Magnetic resonance imaging-based volumetry differentiates progressive supranuclear palsy from corticobasal degeneration. Neuroimage. 2004;21(2):71424.

6 Lieberman AP, Trojanowski JQ, Lee VM, Balin BJ, Ding XS, Greenberg J, et al. Cognitive, neuroimaging, and pathological studies in a patient with Pick's disease. Ann Neurol. 1998;43(2):259-65.

7 Ghetti B, Spina S, Murrell JR, Huey ED, Pietrini $\mathrm{P}$, Sweeney B, et al. In vivo and postmortem clinicoanatomical correlations in frontotemporal dementia and parkinsonism linked to chromosome 17. Neurodegener Dis. 2008; 5(3-4):215-7.

8 Holland D, Brewer JB, Hagler DJ, FennemaNotestine C, Fenema-Notestine C, Dale AM. Subregional neuroanatomical change as a biomarker for Alzheimer's disease. Proc Natl Acad Sci U S A. 2009 Dec;106(49):20954-9.

9 Strain JF, Smith RX, Beaumont H, Roe CM, Gordon BA, Mishra S, et al. Loss of white matter integrity reflects tau accumulation in $\mathrm{Al}-$ zheimer disease defined regions. Neurology. 2018;91(4):E313-8.

10 Thal DR, von Arnim CA, Griffin WS, Mrak RE, Walker L, Attems J, et al. Frontotemporal lobar degeneration FTLD-tau: preclinical lesions, vascular, and Alzheimer-related co-pathologies. J Neural Transm. 2015;122(7): 1007-18.

11 Klohs J, Rudin M. Unveiling molecular events in the brain by noninvasive imaging. Neuroscientist. 2011;17(5):539.

12 Wang N, Zhang J, Cofer G, Qi Y, Anderson RJ, White LE, et al. Neurite orientation dispersion and density imaging of mouse brain microstructure. Brain Struct Funct. 2019; 224(5):1797-813.

13 Wang N, White LE, Qi Y, Cofer G, Johnson GA. Cytoarchitecture of the mouse brain by high resolution diffusion magnetic resonance imaging. Neuroimage. 2020;216(April): 116876.
14 Alexander AL, Lee JE, Lazar M, Field AS. Diffusion tensor imaging of the brain. Neurotherapeutics. 2007 Jul;4(3):316-29.

15 Takahashi S, Yonezawa H, Takahashi J, Kudo $\mathrm{M}$, Inoue $\mathrm{T}$, Tohgi $\mathrm{H}$. Selective reduction of diffusion anisotropy in white matter of $\mathrm{Al}$ zheimer disease brains measured by 3.0 Tesla magnetic resonance imaging. Neurosci Lett. 2002;332(1):45-8.

16 Grandjean J, Schroeter A, He P, Tanadini M, Keist R, Krstic D, et al. Early alterations in functional connectivity and white matter structure in a transgenic mouse model of cerebral amyloidosis. J Neurosci. 2014;34(41):13780.

17 Zhang Y, Schuff N, Du AT, Rosen HJ, Kramer JH, Gorno-Tempini ML, et al. White matter damage in frontotemporal dementia and Alzheimer's disease measured by diffusion MRI. Brain. 2009;132(Pt 9):2579-92.

18 Salat DH, Tuch DS, van der Kouwe AJ, Greve DN, Pappu V, Lee SY, et al. White matter pathology isolates the hippocampal formation in Alzheimer's disease. Neurobiol Aging. 2010 Feb;31(2):244-56.

19 Agosta F, Scola E, Canu E, Marcone A, Magnani $G$, Sarro L, et al. White matter damage in frontotemporal lobar degeneration spectrum. Neurology. 2018;90(12).

20 Zhang Y, Tartaglia MC, Schuff N, Chiang GC, Ching C, Rosen HJ, et al. MRI signatures of brain macrostructural atrophy and microstructural degradation in frontotemporal lobar degeneration subtypes. J Alzheimers Dis. 2012 Dec;33(2):431-44.

21 Agosta F, Galantucci S, Svetel M, Lukić MJ Copetti M, Davidovic K, et al. Clinical, cognitive, and behavioural correlates of white matter damage in progressive supranuclear palsy. J Neurol. 2014;261(5):913-24.

22 Mahoney CJ, Simpson IJ, Nicholas JM, Fletcher PD, Downey LE, Golden HL, et al. Longitudinal diffusion tensor imaging in Frontotemporal dementia. Ann Neurol. 2015; 77(1):33-46.

23 Kantarci K, Murray ME, Schwarz CG, Reid RI, Przybelski SA, Lesnick T, et al. Whitematter integrity on DTI and the pathologic staging of Alzheimer's disease. Neurobiol Aging. 2017;56:172-9.

24 Müller MJ, Greverus D, Dellani PR, Weibrich C, Wille PR, Scheurich A, et al. Functional implications of hippocampal volume and diffusivity in mild cognitive impairment. Neuroimage. 2005;28(4):1033-42.

25 Rose SE, Janke AL, Chalk JB. Gray and white matter changes in Alzheimer's disease: a diffusion tensor imaging study. J Magn Reson Imaging. 2008;27(1):20-6.

26 Strang KH, Golde TE, Giasson BI. MAPT mutations, tauopathy, and mechanisms of neurodegeneration. Lab Invest. 2019;99(7):91228.
27 Yoshiyama Y, Higuchi M, Zhang B, Huang SM, Iwata N, Saido TC, et al. Synapse Loss and microglial activation precede tangles in a P301S tauopathy mouse model. Neuron. 2007;53(3):337-51.

28 Götz J, Chen F, Van Dorpe J, Nitsch RM. Formation of neurofibrillary tangles in P301l tau transgenic mice induced by Abeta 42 fibrils. Science. 2001;293(5534):1491-5.

29 Götz J, Chen F, Barmettler R, Nitsch RM. Tau filament formation in transgenic mice expressing P301L tau. J Biol Chem. 2001;276(1): 529-34.

30 Ramsden M, Kotilinek L, Forster C, Paulson J, McGowan E, SantaCruz K, et al. Age-dependent neurofibrillary tangle formation, neuron loss, and memory impairment in a mouse model of human tauopathy (P301L). J Neurosci. 2005;25(46):10637-47.

31 Santacruz K, Lewis J, Spires T, Paulson J, Kotilinek L, Ingelsson M, et al. Medicine: Tau suppression in a neurodegenerative mouse model improves memory function. Science. 2005;309(5733):476-81.

32 Ni R, Ji B, Ono M, Sahara N, Zhang MR, Aoki $\mathrm{I}$, et al. Comparative in vitro and in vivo quantifications of pathologic tau deposits and their association with neurodegeneration in tauopathy mouse models. J Nucl Med. 2018;59(6): 960-6.

33 Sahara N, Perez PD, Lin WL, Dickson DW, Ren $\mathrm{Y}$, Zeng $\mathrm{H}$, et al. Age-related decline in white matter integrity in a mouse model of tauopathy: An invivo diffusion tensor magnetic resonance imaging study. Neurobiol Aging. 2014;35(6):1364-74

34 Wells JA, O'Callaghan JM, Holmes HE, Powell NM, Johnson RA, Siow B, et al. In vivo imaging of tau pathology using multi-parametric quantitative MRI. Neuroimage. 2015;111: 369-78.

35 Holmes HE, Colgan N, Ismail O, Ma D, Powell NM, O'Callaghan JM, et al. Imaging the accumulation and suppression of tau pathology using multiparametric MRI. Neurobiol Aging. 2016;39:184-94.

36 Colgan N, Siow B, O'Callaghan JM, Harrison IF, Wells JA, Holmes HE, et al. Application of neurite orientation dispersion and density imaging (NODDI) to a tau pathology model of Alzheimer's disease. Neuroimage. 2016; 125:739-44.

37 O'Callaghan J, Holmes H, Powell N, Wells JA, Ismail $\mathrm{O}$, Harrison IF, et al. Tissue magnetic susceptibility mapping as a marker of tau pathology in Alzheimer's disease. Neuroimage. 2017;159(March):334-45.

38 Ni R, Zarb Y, Kuhn GA, Müller R, Yundung Y, Nitsch RM, et al. SWI and phase imaging reveal intracranial calcifications in the P301L mouse model of human tauopathy. Magn Reson Mater Phys. 2020;33(6):769-81. 
39 Saskia Hübner N, Mechling AE, Lee HL, Reisert M, Bienert T, Hennig J, et al. The connectomics of brain demyelination: functional and structural patterns in the cuprizone mouse model. Neuroimage. 2017;146(February 2016):1-18

40 Egger K, Janz P, Döbrössy MD, Bienert T, Reisert M, Obmann M, et al. Microstructural effects of a neuro-modulating drug evaluated by diffusion tensor imaging. Neuroimage. 2016; 127:1-10.

41 Fookes C, Bennamoun M. The use of mutual information for rigid medical image registration: a review. Proc IEEE Int Conf Syst Man Cybern. 2002;4(2002):689-94.

42 Baltes C, Radzwill N, Bosshard S, Marek D, Rudin M. Micro MRI of the mouse brain using a novel $400 \mathrm{MHz}$ cryogenic quadrature RF probe. NMR Biomed. 2009;22(8):834-42.

43 Ma D, Holmes HE, Cardoso MJ, Modat M, Harrison IF, Powell NM, et al. Study the longitudinal in vivo and cross-sectional ex vivo brain volume difference for disease progression and treatment effect on mouse model of tauopathy using automated MRI structural parcellation. Front Neurosci. 2019;13(January):1-18.

44 Sun SW, Neil JJ, Liang HF, He YY, Schmidt $\mathrm{RE}, \mathrm{Hsu} \mathrm{CY}$, et al. Formalin fixation alters water diffusion coefficient magnitude but not anisotropy in infarcted brain. Magn Reson Med. 2005;53(6):1447-51.
45 Shatil AS, Uddin MN, Matsuda KM, Figley CR. Quantitative ex vivo MRI changes due to progressive formalin fixation in whole human brain specimens: longitudinal characterization of diffusion, relaxometry, and myelin water fraction measurements at 3T. Front Med. 2018;5(February):31-15.

46 Thelwall PE, Shepherd TM, Stanisz GJ, Blackband SJ. Effects of temperature and aldehyde fixation on tissue water diffusion properties, studied in an erythrocyte ghost tissue model. Magn Reson Med. 2006;56(2):282-9.

47 Tournier JD, Calamante F, Connelly A. Determination of the appropriate $\mathrm{b}$ value and number of gradient directions for high-angular-resolution diffusion-weighted imaging. NMR Biomed. 2013;26(12):1775-86.

48 Snook L, Plewes C, Beaulieu C. Voxel based versus region of interest analysis in diffusion tensor imaging of neurodevelopment. Neuroimage. 2007;34(1):243-52.

49 Abe O, Takao H, Gonoi W, Sasaki H, Murakami M, Kabasawa $\mathrm{H}$, et al. Voxel-based analysis of the diffusion tensor. Neuroradiology. 2010; 52(8):699-710.

50 Ashburner J, Friston KJ. Voxel-based morphometry: the methods. Neuroimage. 2000; 11(6 Pt 1):805-21.

51 Qin YY, Li MW, Zhang S, Zhang Y, Zhao LY, Lei $\mathrm{H}$, et al. In vivo quantitative whole-brain diffusion tensor imaging analysis of APP/PS1 transgenic mice using voxel-based and atlasbased methods. Neuroradiology. 2013 Aug; 55(8):1027-38.
52 Nowrangi MA, Okonkwo O, Lyketsos C, Oishi K, Mori S, Albert M, et al. Atlas-based diffusion tensor imaging correlates of executive function. J Alzheimers Dis. 2015;44(2):58598.

53 Hess A, Hinz R, Keliris GA, Boehm-Sturm P. On the usage of brain atlases in neuroimaging research. Mol Imaging Biol. 2018;20(5):7429.

54 Koch S, Mueller S, Foddis M, Bienert T, von Elverfeldt D, Knab F, et al. Atlas registration for edema-corrected MRI lesion volume in mouse stroke models. J Cereb Blood Flow Metab. 2019;39(2):313-23.

55 Weston PSJ, Simpson IJA, Ryan NS, Ourselin $S$, Fox NC. Diffusion imaging changes in grey matter in Alzheimer's disease: a potential marker of early neurodegeneration. Alzheimer's Res Ther. 2015;7(1):1-8.

56 Klohs J, Rudin M, Shimshek DR, Beckmann $\mathrm{N}$. Imaging of cerebrovascular pathology in animal models of Alzheimer's disease. Front Aging Neurosci. 2014;6:32.

57 Winklewski PJ, Sabisz A, Naumczyk P, Jodzio K, Szurowska E, Szarmach A. Understanding the physiopathology behind axial and radial diffusivity changes-what do we know? Front Neurol. 2018;9(FEB):92.

58 Reisert M, Kellner E, Dhital B, Hennig J, Kiselev VG. Disentangling micro from mesostructure by diffusion MRI: a Bayesian approach. Neuroimage. 2016;147(October 2016):964-75. 